\title{
Lidar-Monocular Surface Reconstruction Using Line Segments
}

\author{
Victor Amblard ${ }^{1}$, Timothy P. Osedach ${ }^{2}$, Arnaud Croux ${ }^{3}$, Andrew Speck ${ }^{3}$ and John J. Leonard ${ }^{4}$
}

\begin{abstract}
Structure from Motion (SfM) often fails to estimate accurate poses in environments that lack suitable visual features. In such cases, the quality of the final 3D mesh, which is contingent on the accuracy of those estimates, is reduced. One way to overcome this problem is to combine data from a monocular camera with that of a LIDAR. This allows fine details and texture to be captured while still accurately representing featureless subjects. However, fusing these two sensor modalities is challenging due to their fundamentally different characteristics. Rather than directly fusing image features and LIDAR points, we propose to leverage common geometric features that are detected in both the LIDAR scans and image data, allowing data from the two sensors to be processed in a higher-level space. In particular, we propose to find correspondences between 3D lines extracted from LIDAR scans and 2D lines detected in images before performing a bundle adjustment to refine poses. We also exploit the detected and optimized line segments to improve the quality of the final mesh. We test our approach on the recently published dataset, Newer College Dataset. We compare the accuracy and the completeness of the 3D mesh to a ground truth obtained with a survey-grade 3D scanner. We show that our method delivers results that are comparable to a state-of-the-art LIDAR survey while not requiring highly accurate ground truth pose estimates.
\end{abstract}

\section{INTRODUCTION}

Dense reconstruction of large scenes such as buildings or outdoor environments are increasingly compelling for a variety of applications including inspection, change detection and 3D asset creation. Survey-grade 3D LIDAR systems are able to produce very accurate reconstructions of environments but are expensive and can be time-consuming to comprehensively cover large areas. On the other hand, reconstructing a 3D scene solely from monocular images presents a different set of challenges. First, a large number of images with significant overlap must be collected for the poses to be accurately recovered. Additionally, the scene must be well-lit and sufficiently rich in visual features. Finally the scene that is reconstructed may not be referenced to an absolute scale. Recently, the fusion of camera imagery and LIDAR scans for 3D reconstruction has been explored as a way to overcome the respective challenges of these sensing modalities [1], [2], [3]. In this approach, camera imagery captures texture, color, and fine details while LIDAR

This work was supported by Schlumberger Technology Corporation and ONR Grants N00014-18-1-2832 and N00014-19-1-2571.

${ }^{1}$ Currently at Mines ParisTech; work performed at MIT CSAIL; Email: victor. amblardemines-paristech. fr

${ }^{2}$ Work performed at Schlumberger Doll-Research; Current affiliation is General Dynamics Mission Systems; Email: tim. osedach gmail . com

${ }^{3}$ Schlumberger-Doll Research, Cambridge MA; Email: ACroux, ASpeck@slb.com

${ }^{4}$ MIT CSAIL; Email: jleonardemit.edu

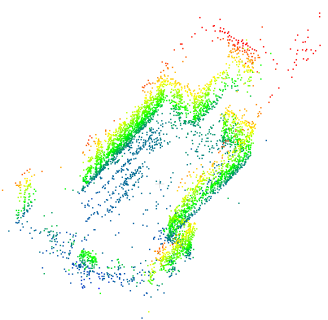

(a) Visual features

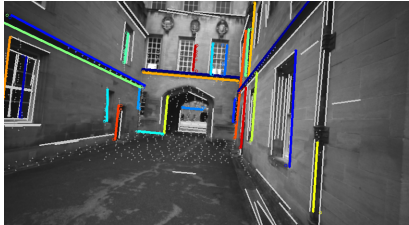

(c) Line extraction

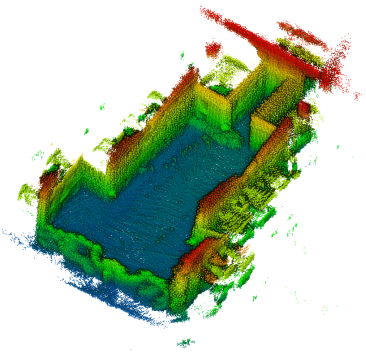

(b) Registered LIDAR scans

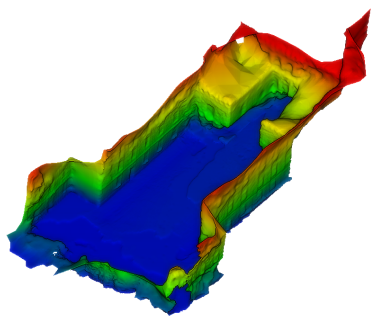

(d) Final 3D reconstruction of the scene
Fig. 1: Our approach, based on lines and points, tightly couples LIDAR scans and monocular images to reconstruct a $3 \mathrm{D}$ scene.

allows accurate range information to be captured that is completely independent of visual complexity (although at a lower resolution). This allows it to fill in gaps where visual feature detection fails. Indeed, the different natures of these sensors make them complementary, but also makes their joint use complex. Semantic or geometric approaches aim to enable processing data from these two different sensor modalities in a higher-level space, leveraging the underlying structure of the environment and reducing the complexity that stems from the modality gap. We focus our work on lines as they are very simple geometric primitives, inexpensive to compute and can be easily found in structured environments. Finding correspondences between 2D lines across different images, however, is a difficult task owing to the depth ambiguity in monocular images. Indeed, two segments whose endpoints are located on the same epipolar line will be reprojected at the same location in images even if their 3D coordinates differ significantly. Therefore, we opt for a 3D line representation that incorporates data from a range sensor, namely LIDAR, along with that from monocular images. These correspondences are then exploited to derive a cost function for bundle adjustment which is then optimized to increase the accuracy of the initial pose 
estimates. The optimized lines and the registered LIDAR scans are then incorporated into a LIDAR-Camera MultiView Stereo (MVS) pipeline. Figure 2 presents an overview of our workflow.

The main contributions of this paper are the following:

- We present a novel end-to-end Structure from Motion algorithm that exploits joint LIDAR/camera observations to derive 3D line segments for localization as well as reconstruction.

- We experimentally validate our approach by testing it on a public dataset.

The remainder of the paper is organized as follows: in section II, we discuss related work in the field of LIDAR-camera fusion for dense reconstruction and SfM using geometric constraints, namely line segments. In section III we present our approach to build a surface mesh based on the coregistered line segments, LIDAR scans and camera images. In section IV, we present qualitative and quantitative results of our system applied to public datasets. Finally section V presents concluding remarks and directions for future work.

\section{RELATED WORK}

\section{A. Geometric primitives for bundle adjustment}

State-of-the-art SfM methods such as OpenMVG [4] and Colmap [5] achieve impressive results with monocular images. The use of geometric primitives and more generally, high-level features, has been shown to allow for more efficient computation, although at the cost of more complex algebraic derivation. [6] provides a theoretical framework for the use of lines in a bundle adjustment and derives an SfM pipeline that is entirely based on lines. Along the same lines, [7] incorporates 2D curves in the bundle adjustment formulation. Moreover, several implementations of Simultaneous Localization and Mapping (SLAM) approaches have been reported that are based in part or entirely on lines or other geometric primitives (for example PL-SLAM [8]). [9] proposes a SLAM algorithm that is based on planes extracted from an RGB-D camera. Similarly, [10] employs structural regularities, such as planes to improve a visualinertial odometry pipeline. [11] uses a prior LIDAR map from which 3D lines are extracted and further used to find correspondences with 2D lines extracted from videos. Our approach, while also using line correspondences to enhance a bundle adjustment, does not require the use of a prior LIDAR map and directly creates 3D segments using the LIDAR data along with the camera data.

\section{B. Lidar-camera fusion}

The robustness of SfM pipelines, however, can be compromised in low-visibility environments (e.g. poor lighting, lack of reliable visual features, etc.) or if the field of view of the camera is narrow. Numerous recent SLAM systems including [12], [13] and [14] make use of a LIDAR along with a camera to overcome this problem. However, the camera data in these implementations is only used to improve localization, or texture a map that is built with LIDAR scans. Conversely, the LIDAR may only be used to add depth information to images. [3] is, to our knowledge, the first work that leverages camera and LIDAR data for dense reconstruction and extends a Multi-View Stereo (MVS) pipeline to utilize LIDAR points during depth map estimation and surface reconstruction. Although related to our work, it initially assumes that poses are accurately known in order to consistently fuse data from those two sensors. Other approaches [1], [2] introduce a probabilistic framework for a joint optimization based on LIDAR patches and points from stereo images. Our work, albeit similar to the recent work from [15], which also presents a framework dealing with lines and exploiting information from LIDAR scans, is directly based on correspondences between 3D line segments. Moreover, since we focus on a Structure from Motion approach, we do not just optimize on a set of recent images but on all images, making robust data association more challenging. To address this, our approach leverages [16], a multi-view data association framework to check the consistency of line segment data associations.

\section{METHODS}

\section{A. Overview}

Before delving into the description of the algorithmic pipeline, we introduce some notation and definitions that will be used throughout the paper.

We consider a set of $N$ images $\left\{I_{i}\right\}_{i \in \mathcal{I}}$ and LIDAR scans $\left\{S_{i}\right\}_{i \in \mathcal{I}}$. The set of camera poses is denoted by $\mathcal{P}$ and the camera pose corresponding to the $i$-th view is $\mathbf{P}_{\mathbf{i}}$.

The projection function $\pi^{i}$ projects a point from the world frame to the $i$-th image plane such that if $\mathbf{X}$ is a 3D point in homogeneous coordinates, and $x$ its $2 \mathrm{D}$ projection onto the image $I_{i}$,

$$
x=\pi^{i}(\mathbf{X})=\mathbf{K} \mathbf{P}_{\mathbf{i}} \mathbf{X}
$$

where $\mathbf{K}$ represents the intrinsic parameters matrix. A 3D infinite line, can be represented by its Plücker coordinates $\mathbf{L} \in \mathbb{R}^{6}$ - or equivalently its Plücker matrix -, and a finite line, l, by its two 3D endpoints. With a slight modification of notation, we define the 3D line segment's projection as being the projection of its two endpoints from the world frame to the image $i$ frame such that $\pi^{i}(\mathbf{l})=$ $\left(\pi^{i}\left({ }^{s} \mathbf{l}\right), \pi^{i}\left({ }^{e} \mathbf{l}\right)\right)$ where ${ }^{s} \mathbf{l}$ represents the starting point of the 3D line and ${ }^{e} \mathbf{l}$ its end point. In the case of an infinite line $\pi^{i}(\mathbf{L})$ represents the three coefficients of the 2D line's equation corresponding to the projection of the 3D infinite line on the $i$-th image. $\mathcal{X}$ represents the set of 3D landmarks used for SfM and the set of observations associated to a 3D landmark $\mathbf{X} \in \mathcal{X}$ is referred to as $\mathcal{O}_{\mathbf{X}}$.

We assume that we have initial estimates of the camera poses that can be obtained for example with a monocular visualinertial SLAM system like VINS [17]. Those priors will help fuse the LIDAR data by (1) providing relative rotations and translations between the camera viewpoints and (2) providing the scale of the scene.

As illustrated in Fig.2, our pipeline includes two components. The first is an enhanced Structure from Motion component which processes on 3D lines, previously detected by a line 


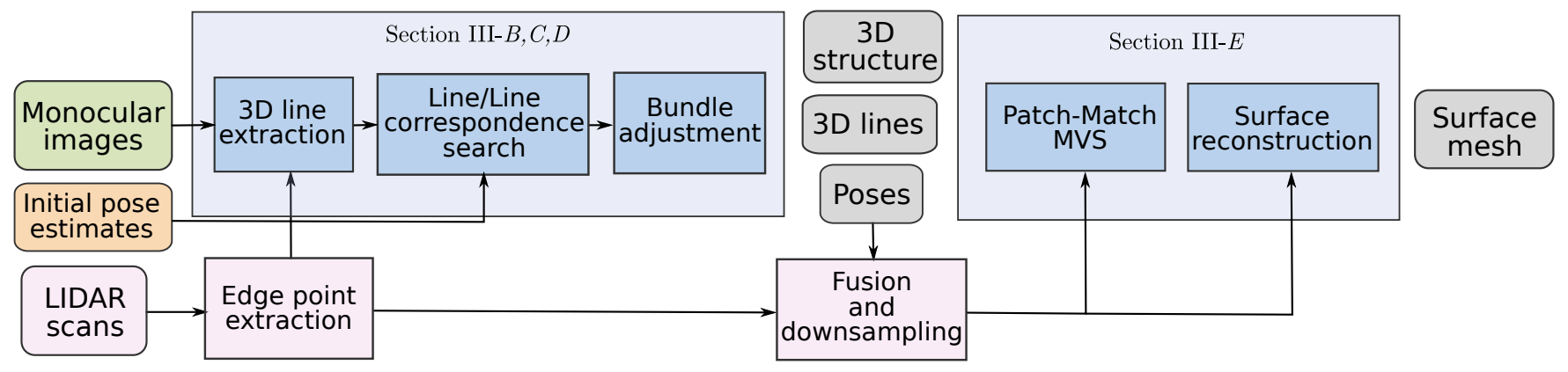

Fig. 2: Overview of our LIDAR-camera reconstruction pipeline

detection module. The line detection module processes data from the LIDAR and the camera to extract and merge 3D lines in the different views. Using correspondence search we are then able to create clusters of lines to yield an additional error term in the bundle adjustment's cost function. Optimization over the new cost function yields new pose estimates. The second component leverages the new pose estimates and the 3D segments to reconstruct a surface mesh that is based on combined information from images and LIDAR scans.

\section{B. Line detection}

The line detection module aims to extract 3D lines that are observed by the camera as well as by the LIDAR.

We begin by identifying potential 2D line segments in images which will constitute the basis to derive the subsequent 3D lines. Line Segment Detector (LSD) [18] is a widely used method that accurately detects line segments based on line support regions. We use this detector to output a set of line segments $\left\{s_{j}^{i}\right\}_{j}$ visible in image $I_{i}$. Then, we augment the $2 \mathrm{D}$ information with range information from the LIDAR (i.e. incorporating a third dimension) and thus to be able to precisely locate the line segment in 3D. Associating the correct LIDAR points to the detected 2D line segments is crucial to the performance of our algorithm. Unlike the system described in [15], which directly associates the closest LIDAR point to the detected 2D line segments, we aim to find LIDAR points that effectively correspond to the observation of the same line. Since LIDAR scans do not provide any texture information, we discard lines corresponding to flat surfaces (i.e. only corresponding to changes in texture), and instead focus the LIDAR/camera association on edges (i.e. lines that correspond to changes in the local surface normal). Hence, in addition to the 2D line segments, we retrieve edge points from LIDAR scans based on a smoothness score as described in [19]. The resulting edge points are then matched to the $2 \mathrm{D}$ segments based on their pixel distance. For each 2D segment $s_{i}^{j}$ we have now associated a set of LIDAR edge points $\left\{e_{j}^{i}\right\}_{j}$. By construction, we know that the selected LIDAR points correspond to a 3D line. However, an ambiguity may remain as to the specific line to which those points correspond. To resolve any ambiguity and ensure that those edge points

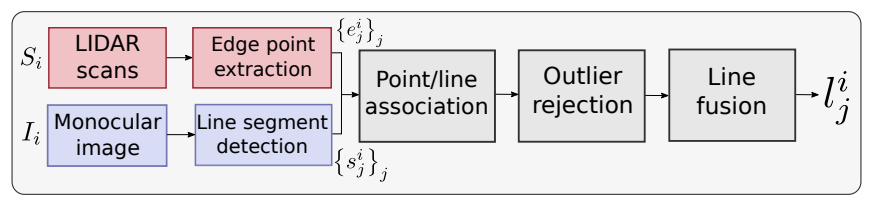

Fig. 3: Overview of the camera/LIDAR 3D segment detection pipeline

correspond to the same line that matches the detected 2D segment, we run RANSAC on the set of edge points. Only inliers are kept and we compare the reprojected 3D line to the 2D segment outputted by LSD. Finally, to avoid multiple instances of the same actual line segment in an image, we also fuse 3D segments having similar directions and which are located on the same infinite line. This procedure is illustrated Fig 3 .

\section{3D/3D line correspondences}

Once 3D segments have been detected across the set of images, we look for segments that represent different views of the same actual segment via 3D/3D correspondences. Let us consider a 3D segment $\mathbf{l}$ detected in the $i$-th image. We first reproject the current line segment on all the images using its 3D information and only keep the views in which the segment is visible. We then iterate over the set of segments detected in those views and compute a similarity score, as described below. Algorithm 1 provides a detailed view of the matching code:

The similarity score $s$ takes into account:

- The angle between the reprojected 3D segments;

- The pixel distance between the projected endpoints;

- The "orthogonal distance" defined as the norm of the vector $\frac{1}{2}\left({ }^{\mathbf{s}} \mathbf{l}+{ }^{\mathbf{e}} \mathbf{l}\right)-\frac{1}{2}\left({ }^{\mathbf{s}} \mathbf{l}^{\prime}+{ }^{\mathbf{e}} \mathbf{l}^{\prime}\right)$ projected perpendicularly to the 3D segment's direction;

- In addition to the three criteria described above, we also use line band descriptors (LBD) [20] which provide additional information about the local appearance of the line.

Each of these four components is weighted by four hyperparameters $\alpha_{\theta}, \alpha_{\text {dist }}, \alpha_{\text {overlap }}, \alpha_{L B D}$ that control the relative importance of the different distances.

Algorithm 1 describes the procedure to compute the set of matches for a single line segment. We repeat the proce- 


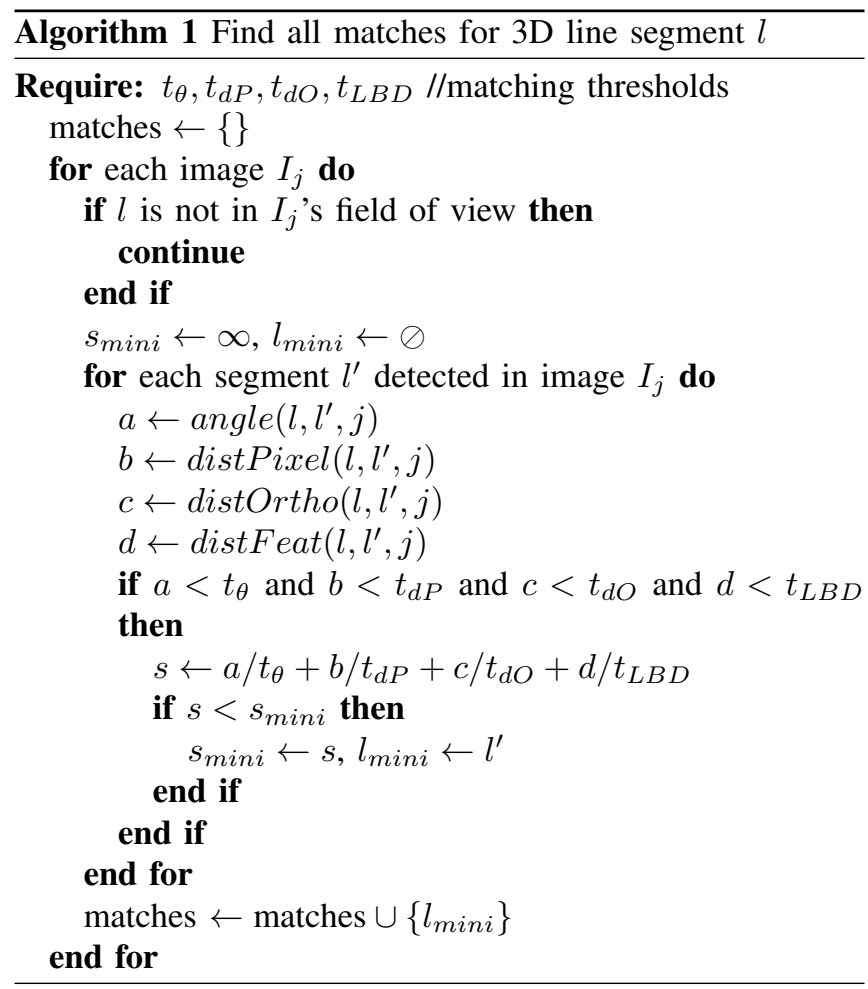

dure for all line segments which eventually yields a set of pairwise associations between line segments. However, some of these associations may not be consistent accross different views, where two line segments from the same view visually representing different actual segments can be connected in the data association graph. To ensure the consistency of the pairwise associations computed during the previous step and to mitigate potentially false data association, we use CLEAR [16].

Based on the filtered pairwise data associations, we infer which line observations $\mathbf{l}_{\mathbf{j}}^{\mathbf{i}}$ correspond to the same actual line and build up a set of lines $\mathcal{L}=\left\{\mathbf{L}_{\mathbf{1}}, \ldots, \mathbf{L}_{\mathbf{m}} \mid m \in \mathbb{N}\right\}$ to which we attach a set $\left\{\mathcal{O}_{\mathbf{L}_{\mathbf{j}}}\right\}$ of line observations where we denote by $\mathcal{O}_{L_{j}}$ the set of observations of line $\mathbf{L}_{\mathbf{j}}$. Because we do not know the exact equation of these lines, we initialize their Plücker vector with an initial rough estimate based on the 3D information provided by their associated observations $\mathcal{O}_{\mathbf{L}_{\mathbf{j}}}$. Lines are further optimized as described in the following paragraph. This process is summarized Fig 4

\section{Line bundle adjustment}

The $M$ 3D lines instances $\mathbf{L}_{1}, \ldots, \mathbf{L}_{\mathbf{M}}$ inferred at the previous step, and their observations allow us to extend the typical bundle adjustment formulation to take into account lines' reprojection errors. Optimizing over finite line segments is often non-trivial because the optimized endpoints may end up in occluded areas. Instead, we represent lines in $\mathcal{L}$ as infinite lines during the optimization step.

The following paragraphs detail the different error terms involved in the cost function that we seek to minimize.

1) Camera observations

We define the point reprojection error of a 3D feature

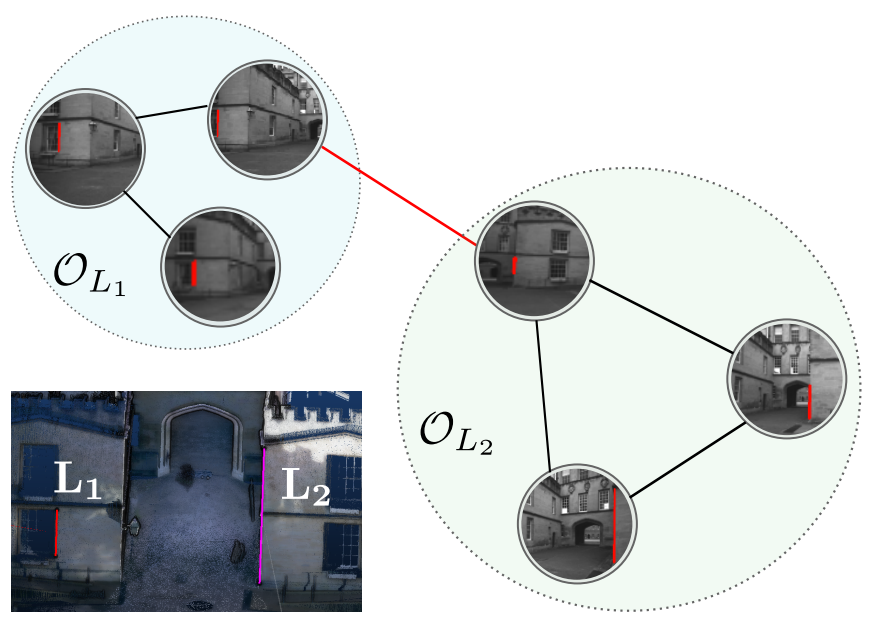

Fig. 4: Pairwise associations (edges) are made following the matching procedure previously described. In red an inconsistent data association filtered out by CLEAR [16] . $\mathcal{O}_{\mathbf{L}_{1}}$ and $\mathcal{O}_{\mathbf{L}_{2}}$ are sets of $3 \mathrm{D}$ line segments $\mathrm{l}_{\mathbf{j}}^{\mathbf{i}}$ from different views but representing the same segment (here resp. $\mathbf{L}_{\mathbf{1}}$ and $\mathbf{L}_{2}$ ) whose accurate location is as yet unknown.

$\mathbf{X}_{\mathbf{j}} \in \mathcal{X}$ as the sum over its observations $\left(x_{k}^{i} \in \mathcal{O}_{\mathbf{X}_{\mathbf{j}}}\right)$ of the pixel distance between its reprojection onto the image plane and its associated observation for that image:

$$
e_{R}\left(\mathbf{X}_{\mathbf{j}}\right)=\sum_{i \in \mathcal{I}} \sum_{x_{k}^{i} \in \mathcal{O}_{\mathbf{X}_{\mathbf{j}}}}\left\|\pi^{i}\left(\mathbf{X}_{\mathbf{j}}\right)-x_{k}^{i}\right\|^{2}
$$

2) Line reprojection:

The line reprojection error of a line $\mathbf{L}_{\mathbf{j}} \in \mathcal{L}$ is defined as being the sum over all its observations of the distances between the observation and the reprojection of line $\mathbf{L}_{\mathbf{j}}$ on the $i$-th image i.e. $\pi^{i}\left(\mathbf{L}_{\mathbf{j}}\right)$. Analogously to [8], if we consider an observation $\mathrm{l}_{\mathrm{k}}^{\mathrm{i}}$ detected in the $i$-th image of the line $\mathbf{L}_{\mathbf{j}}$, the error will be defined as the sum of the $2 \mathrm{D}$ distances between the projected starting point $\pi^{i}\left({ }^{\mathbf{s}} \mathbf{l}_{\mathbf{k}}^{\mathbf{i}}\right)$ and the reprojected infinite line $\pi^{i}\left(\mathbf{L}_{\mathbf{k}}\right)$, and between the projected end point of ${ }^{\mathbf{e}} \mathbf{l}_{\mathbf{j}}^{\mathbf{i}}$ and $\pi^{i}\left(\mathbf{L}_{\mathbf{j}}\right)$.

$$
e_{L}\left(\mathbf{L}_{\mathbf{j}}\right)=\sum_{i \in \mathcal{I}} \sum_{\mathbf{l}_{\mathbf{k}}^{\mathbf{i}} \in \mathcal{O}_{\mathbf{L}_{\mathbf{j}}}}\left\|^{\mathbf{s}} \mathbf{l}_{\mathbf{k}}^{\mathbf{i}}, \pi^{i}\left(\mathbf{L}_{\mathbf{j}}\right)\right\|_{2 D}+\left\|{ }^{\mathbf{e}} \mathbf{l}_{\mathbf{k}}^{\mathbf{i}}, \pi^{i}\left(\mathbf{L}_{\mathbf{j}}\right)\right\|_{2 D}
$$

Based on the error terms (1) and (2) introduced above, the line bundle adjustment cost function can be written as follows:

$$
\mathcal{P}^{*}, \mathcal{X}^{*}, \mathcal{L}^{*}=\underset{\mathcal{P}, \mathcal{X}, \mathcal{L}}{\operatorname{argmin}} \lambda_{R} \sum_{\mathbf{X}_{\mathbf{j}} \in \mathcal{X}} e_{R}\left(\mathbf{X}_{\mathbf{j}}\right)+\lambda_{L} \sum_{\mathbf{L}_{\mathbf{k}} \in \mathcal{L}} e_{L}\left(\mathbf{L}_{\mathbf{k}}\right)
$$

where $\lambda_{L}$ and $\lambda_{R}$ are coefficients chosen to change the relative importance of line factors in the optimization and $\mathcal{P}^{*}, \mathcal{X}^{*}, \mathcal{L}^{*}$ represent respectively the optimized 3D poses, the optimized 3D landmark locations and the optimized infinite line equations. The optimization of (3), implemented with the library Ceres [21], is performed using the Levenberg-Marquart algorithm. We resort to the orthonormal representation [22] for lines during the optimization process. 
This is a minimal representation, using only four parameters, as opposed to Plücker coordinates which require six parameters. As a last step for this module, we transform the infinite lines back to line segments.

\section{E. Lidar-enhanced multi-view stereo}

We now present the last step of our pipeline, which exploits further the detected line segments along with LIDAR scans and camera images. Typically an MVS pipeline includes three steps: depth map computation, depth map fusion and mesh reconstruction, as described fig. 2. Our contribution strictly focuses on the first and last steps.

Provided with the poses computed in the previous step, we register all of the LIDAR point clouds onto a single point cloud $S_{\text {fused }}$ and downsample it using a voxel grid. As in [3], we keep track of the number of LIDAR points that have been clustered in the same voxel. This metric represents a measure of the visibility and relative importance of each voxel for subsequent steps. Depth map computation follows the approach described in [3], in that we rely on LIDAR scans to initialize the depth maps. We slightly modify their approach, however, to reduce potential occlusions: Given the registered LIDAR scan $S_{\text {fused }}$ we initialize each depth map's pixel using the depth of a visual feature, if available. Otherwise, we initialize with the range provided by the LIDAR. Instead of handling occlusions at a pixel's scale (by taking the closest to the camera LIDAR measurement in case of multiple LIDAR measurements available for one pixel) as in [3], we extend the verification to a neighborhood. We check the consistency of the pixel's depth with respect to the average depth in the neighborhood. We keep the LIDAR measurement only if the depth is consistent with the local average. After computing depth maps, we fuse them as in [23]. The last step consists in producing a surface mesh based on the fused depth maps and the registered LIDAR scans. We extend the approach described in [3] which ingeniously combines points from the registered LIDAR scans to points from the dense visual feature cloud. In addition to using this combination we make use of the previously detected line segments. We sample points on each line segment and add them as vertices in the Delaunay triangulation.

\section{Results}

We test our approach on a state-of-the-art public dataset, The Newer College Dataset [24], which provides sequences of images and scans of structured (buildings) environments.

\section{A. Newer college dataset}

The Newer College Dataset consists of different sequences recorded using a handheld rig carried through the New College in Oxford. An Ouster OS-1 64 LIDAR and an Intel Real-Sense D435i are mounted on the rig. In addition, ground truth point cloud data is provided for reconstruction using a Leika BLK360 survey scanner, as well as localization data for the Ouster LIDAR that was derived using the Iterative Closest Point algorithm. We test our approach on

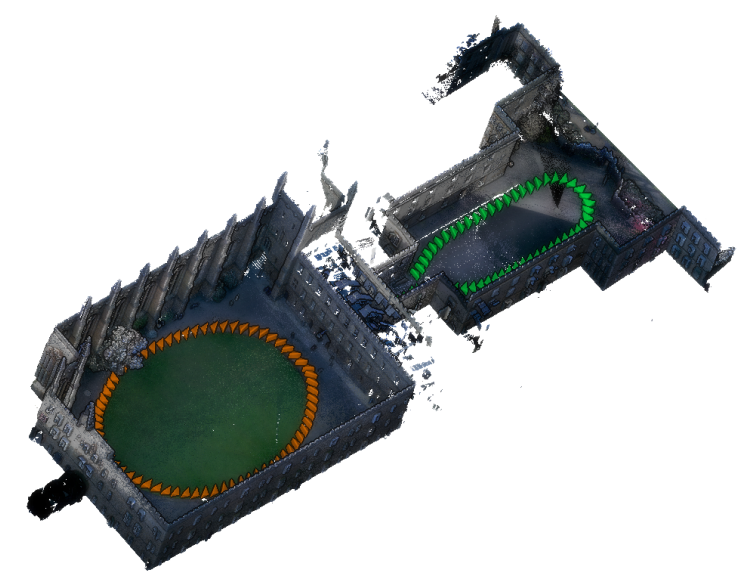

Fig. 5: Ground truth trajectory. The first sequence is displayed in green, the second in orange.

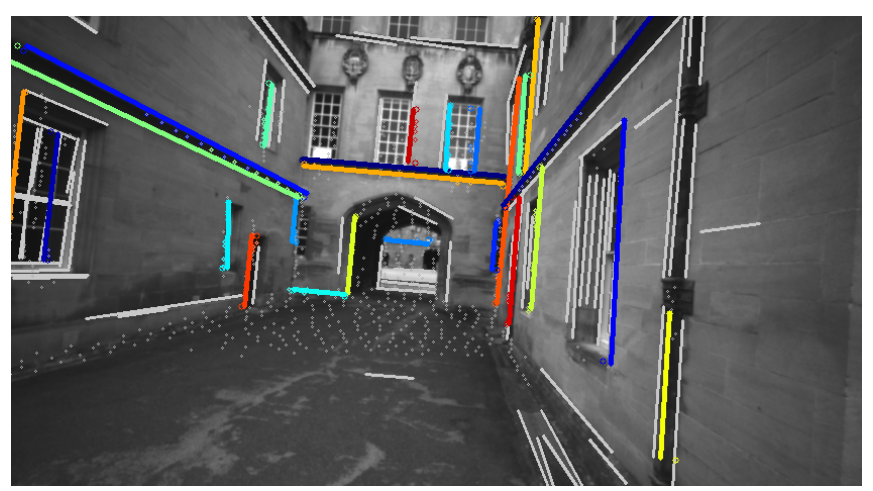

Fig. 6: Each circle represents a detected edge point from the LIDAR scan, lines are detected using a Line Segment detector. The color represents an association between a 2D line segment and edge points. Gray color represents edges and lines that have not been associated

two sequences extracted from the dataset with each containing approximately sixty images. Intrinsic calibration of the camera as well as extrinsic calibration between the camera and the LIDAR are provided. Figure 5 shows the camera's positions in our sequence and a 3D view of the dataset. We generate noisy initial pose estimates by adding random translational $\left(\Sigma_{T}=0.5 \mathrm{~m}\right)$ and rotational noise $\left(\Sigma_{R}=5^{\circ}\right)$ to the ground truth trajectory, but this could also be data provided by an IMU for example.

\section{B. Evaluation}

All experiments were run on an Intel Core i7-8850 CPU with 16GB of RAM. We first evaluate our line-bundle adjustment against a state-of-the art SfM approach [4] using average localization error, a metric which characterizes the accuracy of the estimated camera positions. Since SfM does not compute poses at the metric scale we estimate the unknown scale factor along with the $S E(3)$ transformation matrix to the world frame using Umeyama alignment with the initial noisy poses as an input. Our approach, in constrast, directly utilizes data at metric scale provided by the LIDAR 


\begin{tabular}{ccccc}
\hline \multirow{2}{*}{ Metric } & \multicolumn{2}{c}{ Newer College \#1 } & \multicolumn{2}{c}{ Newer College \#2 } \\
& SfM [4] & Ours & SfM [4] & Ours \\
\hline Avg. localization error (m.) & 0.142 & $\mathbf{0 . 1 4 0}$ & 0.130 & $\mathbf{0 . 1 2 9}$
\end{tabular}

TABLE I: Numerical evaluation of our approach on The Newer College Dataset. Best result shown in bold.

\begin{tabular}{ccccc}
\hline Metric & $\begin{array}{c}\text { Photogrammetry } \\
{[4],[25]}\end{array}$ & $\begin{array}{c}\text { LIDAR only } \\
{[26]}\end{array}$ & $\begin{array}{c}\text { Z. Li et al. } \\
{[3]}\end{array}$ & Ours \\
\hline Precision & $\mathbf{0 . 8 5} / \mathbf{0 . 9 7}$ & $0.77 / 0.90$ & $0.82 / 0.92$ & $0.81 / 0.93$ \\
Recall & $0.45 / 0.84$ & $\mathbf{0 . 7 5 / 0 . 9 7}$ & $0.69 / 0.93$ & $0.69 / 0.93$ \\
F-Score & $0.59 / 0.90$ & $\mathbf{0 . 7 6 / 0 . 9 3}$ & $0.75 / \mathbf{0 . 9 3}$ & $0.74 / \mathbf{0 . 9 3}$
\end{tabular}

TABLE II: Reconstruction results for the Newer College Dataset sequence \#1/sequence \#2. Best result shown in bold

and therefore directly reconstructs the scene at the exact

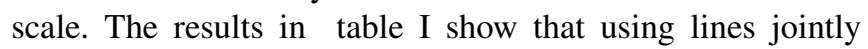
with visual points yields slightly better results than the SfM pipeline for both sequences that were tested. We attribute this improvement to the greater stability that line segments provide relative to point features. This stability derives from the fact that they may remain visible in a more diverse set of views than point features, which may be matched in only a handful of images.

We then evaluate our mesh reconstruction using precision and recall as metrics characterizing both the accuracy of the resulting mesh and its completeness, along with the $F$-score that aggregates both results. As in [3], because our ground truth reconstruction is provided as a point cloud, we first need to transform our mesh to a point cloud and remove occluded areas before comparing it to ground truth. We set the distance threshold to $0.5 \mathrm{~m}$ to compute precision and recall. The main evaluation results, obtained and visualized using the software CloudCompare, are summarized in table III Our algorithm is compared to three other pipelines: a photogrammetry pipeline based on the open source libraries OpenMVG [4] and OpenMVS [25], a LIDAR-only surface reconstruction algorithm [26] based on the ground truth poses, and the approach described in [3]. Illustrations of the final meshes obtained by the different approaches are shown fig. 7

It is evident that photogrammetry results suffer from a lack of camera coverage in some areas and sometimes does not properly render featureless parts of the environment, including some walls and parts of the ground. In contrast, LIDAR-based approaches cover a significantly larger area, but at the cost of precision. As expected, the best overall performance is realized with the LIDAR-only approach, which relies on the highly accurate ground truth poses from the Newer College Dataset. Notably, our approach, which does not require accurate initial poses unlike the one in [3] (as previously discussed), exhibits an F-score that is better than that of photogrammetry and comparable to the LIDARonly case for both test sequences. Our approach is also noticeably more precise in areas where 3D line segments have indeed been detected. Finally, we note that our approach

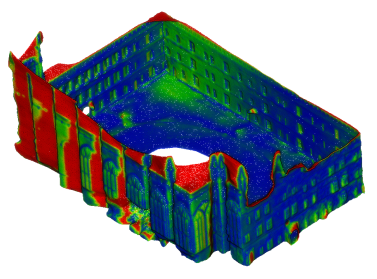

(a) Photogrammetry [4], |25]

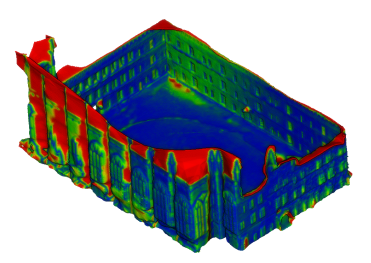

(c) Z.Li et al. [3]

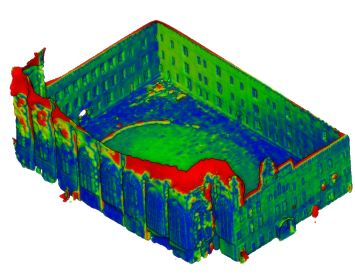

(b) LIDAR only [26]

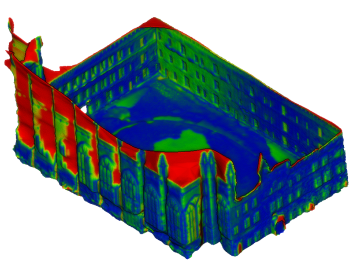

(d) Ours
0

Fig. 7: Surface meshes obtained with different pipelines on sequence \#2. False colors represent the distance of the mesh to ground truth point cloud from blue (close to 0) to red (more than $0.5 \mathrm{~m}$ )

is more applicable to larger scenes since it does not rely on having access to a dense LIDAR point cloud of the environment for depth map initialization.

\section{CONCLUSION AND FUTURE WORK}

We have presented a novel pipeline for LIDAR and camera-based surface reconstruction that is based on 3D line segments: geometric primitives that are computationally inexpensive to obtain, lightweight, and which embed information from both sensors. Starting from noisy pose and line estimates and leveraging state-of-the-art data association algorithms, we are able to exploit optimized line segments and poses to realize an improved a multi-view stereo pipeline. The synthesis of accurate visual features from the camera, the dense - but less accurate - LIDAR point clouds, and the rigid structure information provided by line segments enables a higher quality surface reconstruction than a camera-only approach and comparable performance to a gold standard LIDAR-only 3D reconstruction that relies on highly accurate ground truth pose estimates.

One of the critical assumptions of this work is that the environment be of a structured nature. This requirement makes it appropriate in urban settings. Future work may explore other primitives that could be used jointly with lines to improve performance and relax the assumption of a highly structured environment. [27], for example, presents an interesting approach to leverage $2 \mathrm{D}$ curves during reconstruction to allow recovery of finer details.

\section{ACKNOWLEDGEMENTS}

The authors wish to acknowledge Stéphane Vannuffelen, Sepand Ossia, and Kevin Doherty for insightful discussions and guidance. 


\section{REFERENCES}

[1] W. Zhen, Y. Hu, H. Yu, and S. Scherer. "LiDARenhanced Structure-from-Motion". In: 2020 IEEE International Conference on Robotics and Automation (ICRA). 2020, pp. 6773-6779.

[2] Weikun Zhen, Yaoyu Hu, Jingfeng Liu, and Sebastian Scherer. "A Joint Optimization Approach of LiDAR-Camera Fusion for Accurate Dense 3-D Reconstructions". In: IEEE Robotics and Automation Letters 4.4 (2019), p. 3585.

[3] Zimo Li, Prakruti C. Gogia, and Michael Kaess. "Dense surface reconstruction from monocular vision and LiDAR". In: Proceedings of the IEEE International Conference on Robotics and Automation (ICRA) 2019-May (2019), pp. 6905-6911.

[4] Pierre Moulon, Pascal Monasse, Romuald Perrot, and Renaud Marlet. OpenMVG: Open multiple view geometry. Tech. rep. 2017, pp. 60-74. URL: https : / / pix4d. $\mathrm{com} /$

[5] Johannes Lutz Schönberger and Jan-Michael Frahm. "Structure-from-Motion Revisited". In: Proceedings of the IEEE Conference on Computer Vision and Pattern Recognition (CVPR). 2016.

[6] Adrien Bartoli and Peter Sturm. "Structure-from-Motion Using Lines: Representation, Triangulation, and Bundle Adjustment". In: Computer Vision and Image Understanding (CVIU) 100.3 (Dec. 2005), pp. 416-441.

[7] I. Nurutdinova and A. Fitzgibbon. "Towards Pointless Structure from Motion: 3D Reconstruction and Camera Parameters from General 3D Curves". In: Proceedings of the IEEE International Conference on Computer Vision (ICCV). 2015, pp. 2363-2371.

[8] A. Pumarola, A. Vakhitov, A. Agudo, A. Sanfeliu, and F. Moreno-Noguer. "PL-SLAM: Real-Time Monocular Visual SLAM with Points and Lines". In: Proceedings of the IEEE International Conference on Robotics and Automation (ICRA). 2017.

[9] M. Kaess. "Simultaneous localization and mapping with infinite planes". In: Proceedings of the IEEE International Conference on Robotics and Automation (ICRA). 2015, pp. 4605-4611.

[10] A. Rosinol, T. Sattler, M. Pollefeys, and L. Carlone. "Incremental Visual-Inertial 3D Mesh Generation with Structural Regularities". In: Proceedings of the IEEE International Conference on Robotics and Automation (ICRA). 2019.

[11] H. Yu, W. Zhen, W. Yang, J. Zhang, and S. Scherer. "Monocular Camera Localization in Prior LiDAR Maps with 2D-3D Line Correspondences". In: Proceedings of the IEEE/RSJ International Conference on Intelligent Robots and Systems (IROS). 2020.

[12] J. Zhang and S. Singh. "Visual-lidar odometry and mapping: low-drift, robust, and fast". In: Proceedings of the IEEE International Conference on Robotics and Automation (ICRA). 2015, pp. 2174-2181.

[13] Johannes Graeter, Alexander Wilczynski, and Martin Lauer. "LIMO: Lidar-Monocular Visual Odometry". In: Proceed- ings of the IEEE/RSJ International Conference on Intelligent Robots and Systems (IROS). IEEE. 2018, pp. 7872-7879.

[14] Y. Shin, Y. S. Park, and A. Kim. "Direct Visual SLAM Using Sparse Depth for Camera-LiDAR System”. In: Proceedings of the IEEE International Conference on Robotics and Automation (ICRA). 2018, pp. 5144-5151.

[15] Shi-Sheng Huang, Zeyu Ma, Tai-Jiang Mu, Hongbo Fu, and $\mathrm{S}$. Hu. "Lidar-Monocular Visual Odometry using Point and Line Features". In: 2020.

[16] Kaveh Fathian, Kasra Khosoussi, Yulun Tian, Parker Lusk, and Jonathan P How. "Clear: A consistent lifting, embedding, and alignment rectification algorithm for multiview data association". In: IEEE Transactions on Robotics (2020).

[17] T. Qin, P. Li, and S. Shen. "VINS-Mono: A Robust and Versatile Monocular Visual-Inertial State Estimator". In: IEEE Transactions on Robotics 34.4 (2018), pp. 1004-1020.

[18] Rafael Grompone Von Gioi, Jérémie Jakubowicz, JeanMichel Morel, and Gregory Randall. "LSD: a Line Segment Detector". In: Image Processing On Line 2 (2012), pp. 3555.

[19] T. Shan and B. Englot. "LeGO-LOAM: Lightweight and Ground-Optimized Lidar Odometry and Mapping on Variable Terrain". In: Proceedings of the IEEE/RSJ International Conference on Intelligent Robots and Systems (IROS). 2018, pp. 4758-4765.

[20] Lilian Zhang and Reinhard Koch. "An Efficient and Robust Line Segment Matching Approach Based on LBD Descriptor and Pairwise Geometric Consistency". In: J. Vis. Comun. Image Represent. (Oct. 2013), pp. 794-805.

[21] Sameer Agarwal, Keir Mierle, et al. Ceres Solver. http: //ceres-solver.org

[22] Bartoli and Sturm. "Multiple-view structure and motion from line correspondences". In: Proceedings Ninth IEEE International Conference on Computer Vision. 2003, 207-212 vol.1.

[23] S. Shen. "Accurate Multiple View 3D Reconstruction Using Patch-Based Stereo for Large-Scale Scenes". In: IEEE Transactions on Image Processing 22.5 (2013), pp. 19011914.

[24] M. Ramezani, Y. Wang, M. Camurri, D. Wisth, M. Mattamala, and M. Fallon. "The Newer College Dataset: Handheld LiDAR, Inertial and Vision with Ground Truth". In: Proceedings of the IEEE/RSJ International Conference on Intelligent Robots and Systems (IROS). 2020.

[25] Dan Cernea. "OpenMVS: Multi-View Stereo Reconstruction Library". 2020. URL: https://cdcseacave.github. io/openMVS

[26] Misha Kazhdan, Ming Chuang, Szymon Rusinkiewicz, and Hugues Hoppe. "Poisson Surface Reconstruction with Envelope Constraints". In: Computer Graphics Forum 39.5 (Aug. 2020), pp. 173-182.

[27] S. Li, Y. Yao, T. Fang, and L. Quan. "Reconstructing Thin Structures of Manifold Surfaces by Integrating Spatial Curves". In: 2018 IEEE/CVF Conference on Computer Vision and Pattern Recognition. 2018, pp. 2887-2896. 